\title{
Curvas de crescimento do fruto de cafeeiro cv. Obatã IAC 1669-20 em diferentes alinhamentos de plantio
}

\author{
Growth curves of coffee fruits Obatã IAC 1669-20 in different \\ alignments planting
}

\author{
Antonio Ribeiro da Cunha ${ }^{1 *}$; Clovis Alberto Volpe ${ }^{2}$
}

\begin{abstract}
Resumo
O crescimento do fruto do cafeeiro é altamente dependente de fatores fisiológicos e ambientais. Os fatores ambientais que mais interferem na produtividade do cafeeiro durante os estádios fenológicos críticos são as temperaturas mínimas e máximas do ar, as quais são efeitos da radiação solar e dependente da trajetória aparente do sol. Este trabalho correlacionou massa seca versus massa fresca, e massas fresca e seca em função de dias após o florescimento, de fruto de cafeeiro cv. Obatã IAC 1669-20 plantados em três diferentes alinhamentos baseados na trajetória aparente do Sol, em Jaboticabal, SP. Foram três tratamentos, com quatro repetições, num total de doze parcelas, e cada parcela com cem cafeeiros, sendo que cada tratamento correspondeu a um alinhamento de plantio. Os três alinhamentos utilizados não apresentaram diferenças no valor médio de massa fresca e seca. A taxa de crescimento do fruto em massa fresca foi maior na fase exponencial, enquanto que em massa seca, foi maior na fase linear. A massa seca $(M S)$ pode ser estimada a partir da massa fresca $(M F)$ por meio do modelo: $M S=A 1 * \exp (M F / t 1)$. O modelo sigmoidal que representou biologicamente o crescimento do fruto do cafeeiro em massa fresca e/ou seca em função de dias após a florada (DAF) foi: $M F(M S)=A 2+[(A 1-A 2) /(1+\exp (D A F-x 0) / d x)]$.
\end{abstract}

Palavras-chave: Coffea arabica L, massas fresca e seca de fruto, modelagem

\begin{abstract}
The growth of the coffee fruit is highly dependent on physiological and environmental factors. Environmental factors that most influence the yield of coffee during the critical growth stages are the minimum and maximum temperatures of air, which are affected by solar radiation and depend on the apparent path of the sun. This research work correlated dry mass versus fresh mass, and, fresh and dry mass as function of days after flowering of fruit coffee cv. Obatã IAC 1669-20 planted in three different alignments based on the apparent trajectory of the sun, in Jaboticabal, SP. The planting was aligned according to the apparent path of the sun. There were three treatments with four replications, a total of twelve plots, each plot with one hundred plants of coffee, each treatment corresponded to an alignment of planting. The three alignments used showed no differences with respect the average fresh and dry mass. The growth rate of fruit, in fresh weight, was higher in the exponential phase, and in dry mass was higher in the linear stage. The dry weight $(D W)$ can be estimated from the fresh weight $(F W)$ using the model: $D W=A 1 * \exp (F W / t 1)$. The sigmoidal model that represents the fruit growth in of coffee plants in fresh and/or dry mass in terms of days after flowering $(D A F)$ was: $F W(D W)=A 2+[(A 1-A 2) /(1+\exp (D A F-x 0) / d x)]$.
\end{abstract}

Key words: Coffea arabica $\mathrm{L}$, fresh and dry fruit weight, modeling

${ }^{1}$ Eng $^{\circ}$. Agro. Dr., Universidade Estadual Paulista Júlio de Mesquita Filho, Depto. de Recursos Naturais, FCA-UNESP, Cx. Postal 237, CEP 18610-307, Botucatu, SP. E-mail: arcunha@fca.unesp.br

${ }^{2}$ In memorian. Prof. Dr. Adjunto, Depto. de Ciências Exatas, FCAV-UNESP, CEP 14884-900, Jaboticabal, SP.

* Autor para correspondência 


\section{Introdução}

As principais espécies de Coffea no mercado mundial são $C$. arabica, originária da Etiópia, Sudão e Quênia e C. canephora, oriunda de regiões tropicais e subtropicais do continente africano (CHEVALIER, 1947), sendo que C. arabica, conhecida genericamente como café arábico, é responsável por $75 \%$ da produção comercializada e preferida devido à melhor qualidade da bebida.

O setor agrícola cafeeiro no Brasil ocupa aproximadamente 2,1 milhões de hectares plantados com 5,7 milhões de pés de café. Segundo a safra de 2010, o estado de Minas Gerais se destaca apresentando uma área produtiva de 1.006.719 ha, seguido dos estados do Espírito Santo (460.193 ha), São Paulo (167.147 ha), Rondônia (154.879 ha), Bahia (139.550 ha), Paraná (81.874 ha), Mato Grosso (15.186 ha), Pará (13.500 ha) e o Rio de Janeiro (13.100 ha), além de outros estados com menor expressão (24.477 ha) (CONAB, 2010).

Atualmente, a cv. Obatã IAC 1669-20, pertencente à espécie $C$. arabica vem sendo extensivamente plantada por cafeicultores das mais diversas regiões produtoras do país, pois é adaptada ao cultivo adensado e em renque, apresenta porte reduzido, produção elevada e resistência ao fungo Hemileia vastatrix (ferrugem alaranjada), e também com maturação mais tardia que a cultivar Catuaí, o que permite um melhor escalonamento da colheita (FAZUOLI et al., 2000).

A produção do cafeeiro depende das condições climáticas locais e de vários outros fatores (RENA; MAESTRI, 1986, 1987, LAVIOLA et al., 2007), sendo que o crescimento do fruto de cafeeiro passa por cinco estádios distintos de formação: chumbinho, expansão rápida, crescimento suspenso, granação e maturação. Cada estádio de formação possui funções fisiológicas e metabólicas próprias, essenciais à formação final da semente de café (LAVIOLA et al., 2007), e dependem do comportamento fenológico da cultura, e da interação de fatores ambientais (níveis de radiação solar e de temperatura) (MOENS, 1962; CASTILLO; LOPEZ, 1966; WORMER; GITUANJA, 1970) com o suprimento de água (DEAN, 1939; MOENS, 1962).

O crescimento do fruto do cafeeiro é altamente dependente de fatores fisiológicos, tratos culturais e ambientais. Dos fatores ambientais, os elementos temperaturas mínimas e máximas do ar, que são efeitos da radiação solar, interferem na produtividade da cultura durante os estádios fenológicos críticos. Sabendo-se que o sistema de cultivo adensado na linha cria modificações no microclima provocadas pelo maior número de plantas, e que isso altera o seu comportamento do ponto de vista fisiológico, tornam-se necessários estudos que analisem esta nova realidade de sistema de cultivo associado a diferentes alinhamentos. Com isso, este trabalho correlacionou massa seca versus massa fresca, e massas fresca e seca em função de dias após o florescimento, de frutos de cafeeiros cv. Obatã IAC 1669-20 plantados em três diferentes alinhamentos baseados na trajetória aparente do Sol, em Jaboticabal, SP.

\section{Material e Métodos}

$\mathrm{O}$ ensaio foi conduzido próximo à Estação Agroclimatológica pertencente à área de Agrometeorologia do Departamento de Ciências Exatas da Faculdade de Ciências Agrárias e Veterinárias, UNESP, Campus de Jaboticabal, SP (latitude: $21^{\circ} 14^{\prime} 05^{\prime} \mathrm{S}$; longitude: $48^{\circ} 17^{\prime} 09^{\prime \prime} \mathrm{W}$; e altitude: $615 \mathrm{~m}$ ), onde está instalada uma cultura de cafeeiro cv. Obatã IAC 1669-20, com 5 anos de idade, altura média de $2 \mathrm{~m}$ e espaçamento de $3,5 \mathrm{x}$ $0,5 \mathrm{~m}$.

O plantio da lavoura experimental foi feito em 13/07/2001. Foram definidos três alinhamentos de plantio para o cafeeiro em função de três estádios fenológicos: $\left.1^{\circ}\right)$ vegetação plena $(15 / 01)$; $\left.2^{\circ}\right)$ maturação do fruto $(15 / 05)$; e $3^{\circ}$ ) floração e enchimento do grão (15/09), sendo essas épocas definidas com base nos estádios fenológicos 
descritos por Camargo e Camargo (2001). Esses três alinhamentos do cafeeiro foram calculados mediante a determinação da posição do sol em certo instante, num dado local e data, a partir do ângulo zenital e do azimute do sol, sendo que o ângulo zenital é dependente da latitude local, declinação solar e do ângulo horário.

Para os cálculos adotou-se o horário das $9 \mathrm{~h}$, pois o cafeeiro é uma planta $\mathrm{C} 3$, mas comporta-se como uma C4 por apresentar alta produtividade a pleno sol, o que permite o aumento da fotossíntese líquida em função de incremento na irradiância a partir desse horário (JONES, 1994). A partir das 9 h o sol estará numa posição formando um ângulo horário de $45^{\circ}$ com um ponto referencial da Terra para uma dada latitude e data, indicando um deslocamento máximo angular que o Sol pode ter para haver maior disponibilidade de radiação solar naquele ponto.
Como o ângulo zenital é quem determina a posição do sol em relação ao plano vertical local, foi possível a partir do mesmo, determinar o ângulo no plano horizontal entre a projeção do raio do Sol e o ponto cardeal N-S, para calcular os três alinhamentos.

A (Tabela 1) apresenta o ângulo horário, declinação do Sol, ângulo zenital e o azimute, sendo este último que caracteriza o alinhamento para cada uma das datas definidas.

Com isso, foi possível instalar os três alinhamentos de plantio do cafeeiro: $81^{\circ}-261^{\circ}(15 /$ jan), $51^{\circ}-231^{\circ}(15 / \mathrm{mai})$ e $66^{\circ}-246^{\circ}(15 / \mathrm{set})$, sendo que cada alinhamento corresponde ao tratamento com 4 repetições, num total de 12 parcelas, com 100 cafeeiros em cada uma, segundo esquema na (Figura 1).

Tabela 1. Valores obtidos do ângulo horário, declinação do Sol, ângulo zenital e azimute para a localidade de Jaboticabal, SP.

\begin{tabular}{lcccc}
\hline Data & Ângulo horário (h) & Declinação do Sol (d) & Ângulo Zenital (Z) & Azimute (a) \\
\hline 15 de janeiro & $45^{\circ}$ & $21,3^{\circ} \mathrm{S}$ & $41,8^{\circ}$ & $81,4^{\circ}-261,4^{\circ}$ \\
15 de maio & $45^{\circ}$ & $18,7^{\circ} \mathrm{N}$ & $59,5^{\circ}$ & $51^{\circ}-231^{\circ}$ \\
15 de setembro & $45^{\circ}$ & $2,2^{\circ} \mathrm{N}$ & $50,5^{\circ}$ & $66^{\circ}-246^{\circ}$ \\
\hline
\end{tabular}

A plantas de cateeiro

Figura 1. Representação esquemática da distribuição dos alinhamentos, destacando a linha central dos 3 alinhamentos onde foram feitas as medidas das variáveis meteorológicas. 
Em função das anomalias ocorridas nas floradas dos anos agrícolas de 2006/2007 e 2007/2008, e tendo poucos ramos com flor, as coletas de frutos no cafeeiro tiveram que ser feitas aleatoriamente em cada alinhamento dentro das repetições, constando de 100 frutos por alinhamento, num total de 300 frutos em toda área do ensaio para cada uma das datas: 06/11/07 (96 DAF), 21/11/07 (111 DAF), 06/12/07 (126 DAF), 21/12/07 (141 DAF), 07/01/08 (158 DAF), 23/01/08 (174 DAF), 07/02/08 (189 DAF), 22/02/08 (204 DAF), 08/03/08 (219 DAF), 24/03/08 (235 DAF), 08/04/08 (250 DAF), 23/04/08 (265 DAF), 06/05/08 (279 DAF) e 21/05/08 (293 DAF). Foram coletados frutos nas duas faces separadamente, norte $(\mathrm{N})$ e sul $(\mathrm{S})$. O material colhido era acondicionado em sacos plásticos para posterior pesagem em balança analítica de $0,0001 \mathrm{~g}$ de precisão, e assim obter a massa fresca. Após, esse material era colocado em estufa com fluxo de ar a $80{ }^{\circ} \mathrm{C}$ por 72 horas para obter a massa seca.

O tratamento dos dados meteorológicos medidos, ou seja, cálculos matemáticos, confecção de gráficos e ajustes de modelos foram feitos em ambiente "Microcal Origin" (MICROCAL SOFTWARE, INC., 1999). Foram feitos ajustes de modelos para explicar o fenômemo do crescimento do fruto de cafeeiro para obter equações de estimativa para a massa fresca e/ou seca em função dos dias após o florescimento (DAF) e a estimativa da massa seca em função da massa fresca.

Na correlação da massa seca (MS) em função da massa fresca (MF) do fruto de cafeeiro, a equação que melhor ajustou-se ao fenômeno foi a de forma exponencial de crescimento:

$$
y=A 1 * \exp \left(\frac{x}{t 1}\right)
$$

onde $y$ é a massa seca ( $\mathrm{g}$ ) em função da massa fresca $(\mathrm{g}), A 1$ o valor inicial da massa seca $(\mathrm{g}), x$ a massa fresca $(\mathrm{g})$, e $t 1$ o valor máximo de massa seca $(\mathrm{g})$.

$\mathrm{Na}$ correlação entre a massa fresca e/ou seca em função de dias após florescimento (DAF) a equação que melhor ajustou-se ao fenômeno foi a de forma sigmoidal de crescimento de Boltzmann:

$$
y=A 2+\frac{(A 1-A 2)}{\frac{1+\exp (x-x 0)}{d x}}
$$

em que $y$ é a massa fresca ou seca (g), $A 1$ o valor inicial da massa fresca ou seca $(\mathrm{g}), A 2$ o valor final da massa fresca ou seca $(\mathrm{g}), x 0$ o ponto de inflexão, ou seja, valor da ordenada $\mathrm{x}(D A F)$ que corresponde ao valor médio da massa fresca ou seca $(x 0,(A 1+A 2) / 2)$, e o $d x$ o parâmetro de ajuste, ou seja, fator de espalhamento da curva que indica o grau de aumento da massa fresca ou seca em função do aumento de $\mathrm{x}(D A F)$.

\section{Resultados e Discussão}

Nota-se pela (Figura 2) as variações fenológicas que ocorreram no fruto de cafeeiro desde o florescimento até a maturação, para os alinhamentos $81-261^{\circ}, 51-231^{\circ}$ e $66-246^{\circ}$ nas faces $\mathrm{N}$ e $\mathrm{S}$ considerando a matéria fresca (MF) e seca (MS). Esse período foi definido desde os 96 dias após o florescimento (DAF) até os 293 DAF, momento em que ocorreu a maturação do fruto e conseqüente perda d'água. $\mathrm{O}$ alinhamento $81^{\circ}-261^{\circ}$ foi o que apresentou a maior média de massa fresca, 0,64 $\mathrm{g}$ para a face $\mathrm{N}$ e $0,62 \mathrm{~g}$ para a face $\mathrm{S}$. No caso da massa seca, este alinhamento foi superior apenas na face $\mathrm{N}$, com $0,16 \mathrm{~g}$.

É interessante relatar que a cv. Obatã IAC 166920 apresenta aderência dos frutos ao ramo, ou seja, a queda de fruto maduro ou seco é bastante reduzida, sendo isso de fundamental importância para a colheita mecânica do fruto (FAZUOLI et al., 2001).

Nota-se pela curva das massas fresca e seca para as duas faces, $\mathrm{N}$ e $\mathrm{S}$, que o fruto apresentou um crescimento lento no início, dos 96 aos 126 DAF (fase logarítmica), e logo após, um rápido crescimento dos 127 até os 158 DAF (fase exponencial). O fruto continua a crescer de forma menos acelerada, dos 
159 até os 279 DAF (fase linear), ocorrendo logo depois da maturação, momento no qual o fruto paralisa o seu crescimento, e inicia o processo de diminuição do volume e massa do fruto em função da perda d'água (Figura 2).

Na fase de crescimento lento, dos 96 aos 126 DAF (Figura 2), o fruto estava no estádio de chumbinho apresentando uma baixa taxa de crescimento devido à elevada taxa respiratória e multiplicação celular (CANNELL, 1971; LEON; FOURNIER, 1962). Esta fase de crescimento ocorre normalmente entre 15 e 28 dias (RAMAIAH; VASUDEVA, 1969; OYEBADE, 1976; LEON; FOURNIER, 1962; WORMER, 1964), sendo que nesta pesquisa ocorreu em 30 dias. Importante destacar que houve um déficit hídrico acumulado em outubro de $83 \mathrm{~mm}$ (Tabelas 2 e 3), o qual influenciou nesta baixa taxa de crescimento. As chuvas iniciaram em 23/10/07 com baixos índices, e com isso o solo não atingiu sua capacidade de campo. Devido a esses baixos índices pluviométricos, o crescimento do fruto ficou prejudicado, sendo que somente após os 126 DAF os índices pluviométricos começaram a aumentar e suprir essas deficiências hídricas. Isto corrobora com Camargo (1987), o qual afirma que nas condições da região centro-sul do Brasil o déficit hídrico na fase de chumbinho (outubro a dezembro) atrasa o crescimento do fruto e reduz a produtividade.

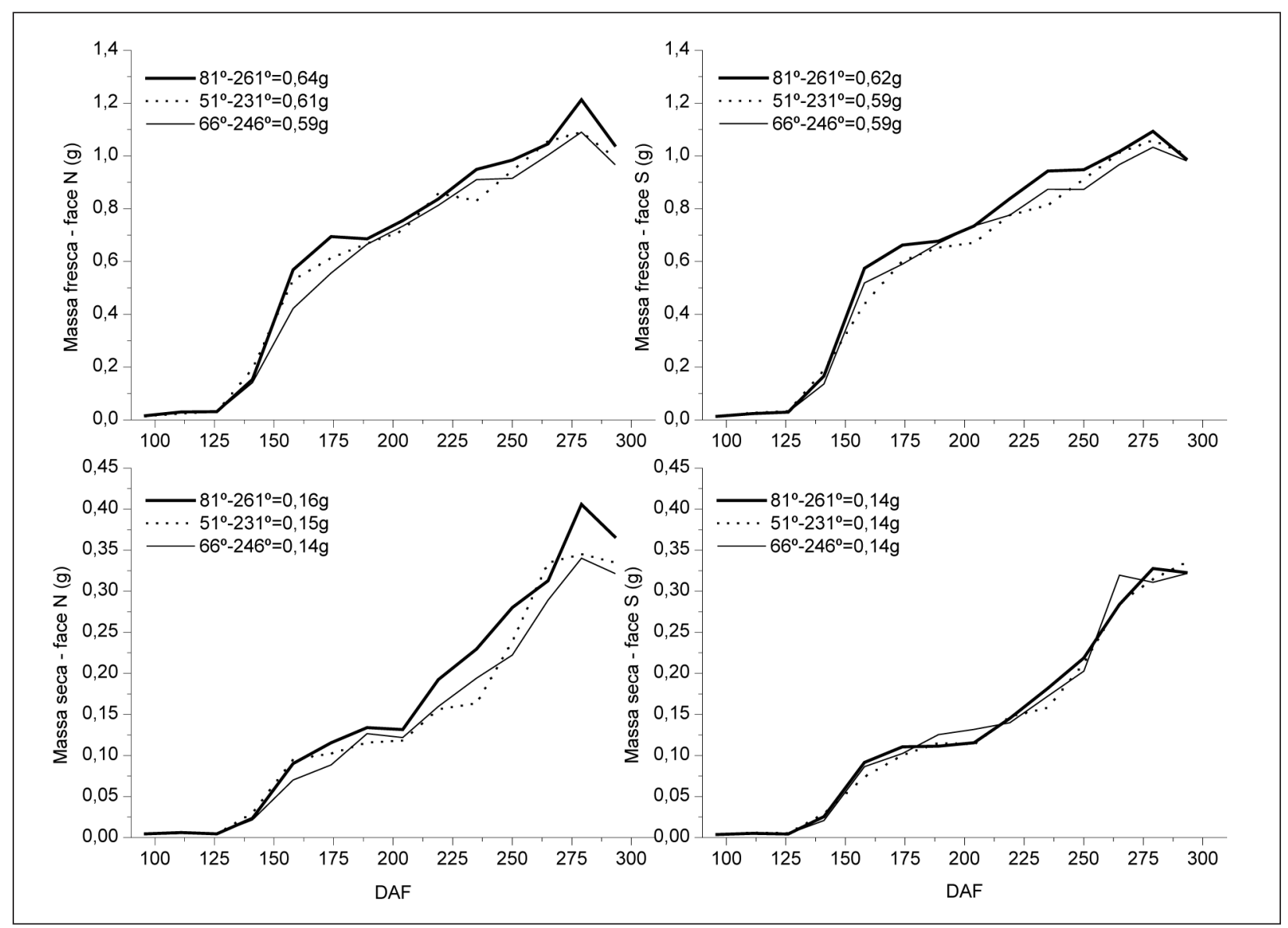

Figura 2. Crescimento do fruto de cafeeiro cv. Obatã IAC 1669-20 nos alinhamentos $81-261^{\circ}, 51-231^{\circ}$ e $66-246^{\circ}$, nas faces $\mathrm{N}$ e $\mathrm{S}$, considerando massas fresca e seca. 
Tabela 2. Massa fresca (g) do fruto de cafeeiro cv. Obatã IAC 1669-20 nos três alinhamentos e nas faces N e S.

\begin{tabular}{|c|c|c|c|c|c|c|c|c|c|c|c|c|}
\hline \multirow{3}{*}{ DAF } & \multicolumn{4}{|c|}{$81^{\circ}-261^{\circ}$} & \multicolumn{4}{|c|}{$51^{\circ}-231^{\circ}$} & \multicolumn{4}{|c|}{$66^{\circ}-246^{\circ}$} \\
\hline & $\begin{array}{l}\text { MF } \\
\text { FN }\end{array}$ & CV & $\begin{array}{c}\text { MF } \\
\text { FS }\end{array}$ & $\begin{array}{l}\text { CV } \\
\%\end{array}$ & MF & CV & $\begin{array}{c}\text { MF } \\
\text { FS }\end{array}$ & CV & MF & CV & $\begin{array}{l}\text { MF } \\
\text { FS }\end{array}$ & $\begin{array}{l}\text { CV } \\
\text { o }\end{array}$ \\
\hline & $\frac{\text { FN }}{0.016}$ & $\frac{\%}{60}$ & FS & $\%$ & FN & $\frac{\%}{31}$ & FS & $\%$ & FN & $\begin{array}{c}\% \\
118\end{array}$ & FS & $\%$ \\
\hline 111 & 0,031 & 14,2 & 0,024 & 10,4 & 0,024 & 14,2 & 0,027 & 10,5 & 0,027 & 18,4 & 0,026 & 21,4 \\
\hline 126 & 0,030 & 8,8 & 0,029 & 8,3 & 0,030 & 12,3 & 0,033 & 24,1 & 0,030 & 17,3 & 0,030 & 28,7 \\
\hline 141 & 0,153 & 22,9 & 0,166 & 15,4 & 0,194 & 14,6 & 0,189 & 16,1 & 0,141 & 12,6 & 0,136 & 27,4 \\
\hline 158 & 0,569 & 11,0 & 0,574 & 19,0 & 0,529 & 9,7 & 0,439 & 15,0 & 0,422 & 15,7 & 0,519 & 10,4 \\
\hline 174 & 0,694 & 8,6 & 0,662 & 18,9 & 0,614 & 23,9 & 0,602 & 22,4 & 0,556 & 18,5 & 0,590 & 5,4 \\
\hline 189 & 0,686 & 10,9 & 0,677 & 11,7 & 0,668 & 2,3 & 0,653 & 5,4 & 0,666 & 3,1 & 0,669 & 11,6 \\
\hline 204 & 0,755 & 1,0 & 0,735 & 6,4 & 0,716 & 8,4 & 0,671 & 4,9 & 0,733 & 11,3 & 0,735 & 7,0 \\
\hline 219 & 0,837 & 8,2 & 0,838 & 4,7 & 0,831 & 3,1 & 0,775 & 6,1 & 0,813 & 6,4 & 0,775 & 6,3 \\
\hline 235 & 0,949 & 5,9 & 0,943 & 0,7 & 0,858 & 2,7 & 0,814 & 1,8 & 0,911 & 4,9 & 0,874 & 6,0 \\
\hline 250 & 0,984 & 1,0 & 0,948 & 14,4 & 0,945 & 4,3 & 0,911 & 2,8 & 0,915 & 6,3 & 0,873 & 3,3 \\
\hline 265 & 1,045 & 1,1 & 1,017 & 1,7 & 1,056 & 3,8 & 1,012 & 1,8 & 1,003 & 4,4 & 0,967 & 6,0 \\
\hline 279 & 1,212 & 2,8 & 1,093 & 5,7 & 1,090 & 11,6 & 1,059 & 3,8 & 1,090 & 1,9 & 1,033 & 3,2 \\
\hline 293 & 1,040 & 3,6 & 0,989 & 2,3 & 0,994 & 6,3 & 1,005 & 1,8 & 0,968 & 8,6 & 0,982 & 1,3 \\
\hline Média & $0,643 a$ & - & $0,622 a$ & - & $0,612 a$ & - & $0,586 a$ & - & $0,592 a$ & - & $0,587 a$ & - \\
\hline
\end{tabular}

$\mathrm{DAF}=$ dia após a florada; $\mathrm{MF}=$ massa fresca; $\mathrm{FN}=$ face norte; $\mathrm{FS}=$ face sul; $\mathrm{CV}=$ coeficiente de variação. Médias seguidas da mesma letra não diferem entre si pelo teste $\mathrm{F}$ ao nível de $1 \%$ de probabilidade.

Tabela 3. Massa seca (g) do fruto de cafeeiro cv. Obatã IAC 1669-20 nos três alinhamentos e nas faces N e S.

\begin{tabular}{|c|c|c|c|c|c|c|c|c|c|c|c|c|}
\hline \multirow{3}{*}{ DAF } & \multicolumn{4}{|c|}{$81^{\circ}-261^{\circ}$} & \multicolumn{4}{|c|}{$51^{\circ}-231^{\circ}$} & \multicolumn{4}{|c|}{$66^{\circ}-246^{\circ}$} \\
\hline & MS & CV & MS & $\mathrm{CV}$ & MS & $\mathrm{CV}$ & MS & $\mathrm{CV}$ & MS & $\mathrm{CV}$ & MS & $\mathrm{CV}$ \\
\hline & FN & $\%$ & FS & $\%$ & FN & $\%$ & FS & $\%$ & FN & $\%$ & FS & $\%$ \\
\hline 96 & 0,005 & 20,7 & 0,004 & 20,5 & 0,004 & 13,8 & 0,004 & 32,5 & 0,004 & 19,4 & 0,004 & 18,4 \\
\hline 111 & 0,005 & 20,9 & 0,005 & 24,8 & 0,005 & 11,2 & 0,006 & 8,2 & 0,006 & 13,7 & 0,006 & 18,0 \\
\hline 126 & 0,006 & 5,4 & 0,004 & 13,3 & 0,005 & 16,3 & 0,006 & 6,0 & 0,005 & 16,6 & 0,005 & 4,6 \\
\hline 141 & 0,024 & 6,4 & 0,026 & 4,1 & 0,030 & 7,6 & 0,029 & 6,5 & 0,022 & 13,8 & 0,021 & 26,0 \\
\hline 158 & 0,090 & 5,2 & 0,092 & 3,4 & 0,095 & 18,9 & 0,074 & 17,2 & 0,070 & 13,4 & 0,086 & 20,9 \\
\hline 174 & 0,116 & 4,4 & 0,110 & 10,5 & 0,102 & 8,6 & 0,100 & 9,1 & 0,089 & 3,3 & 0,102 & 5,8 \\
\hline 189 & 0,134 & 1,0 & 0,111 & 3,7 & 0,116 & 4,3 & 0,115 & 2,8 & 0,127 & 6,3 & 0,125 & 14,2 \\
\hline 204 & 0,132 & 6,3 & 0,116 & 14,4 & 0,118 & 6,0 & 0,114 & 4,9 & 0,122 & 2,2 & 0,132 & 0,4 \\
\hline 219 & 0,192 & 5,0 & 0,145 & 5,5 & 0,163 & 16,6 & 0,147 & 0,9 & 0,160 & 1,5 & 0,140 & 6,0 \\
\hline 235 & 0,230 & 1,1 & 0,182 & 5,0 & 0,157 & 2,5 & 0,158 & 6,7 & 0,194 & 3,6 & 0,172 & 2,2 \\
\hline 250 & 0,280 & 5,2 & 0,219 & 2,7 & 0,239 & 4,1 & 0,212 & 9,4 & 0,222 & 0,4 & 0,203 & 0,3 \\
\hline 265 & 0,313 & 0,4 & 0,284 & 6,3 & 0,335 & 2,7 & 0,286 & 1,8 & 0,289 & 3,4 & 0,320 & 1,4 \\
\hline 279 & 0,406 & 5,7 & 0,328 & 3,0 & 0,345 & 8,3 & 0,315 & 2,4 & 0,340 & 6,2 & 0,311 & 6,9 \\
\hline 293 & 0,366 & 3,2 & 0,323 & 3,0 & 0,335 & 9,6 & 0,335 & 3,9 & 0,322 & 0,5 & 0,322 & 11,3 \\
\hline Média & $0,164 a$ & - & $0,139 a$ & - & $0,146 a$ & - & $0,136 a$ & - & $0,141 \mathrm{a}$ & - & $0,139 a$ & - \\
\hline
\end{tabular}

$\mathrm{DAF}=$ dia após a florada; $\mathrm{MS}=$ massa seca; $\mathrm{FN}=$ face norte; $\mathrm{FS}=$ face sul; $\mathrm{CV}=$ coeficiente de variação. 
Dos 127 aos 158 DAF ocorreu a fase de crescimento rápido devido ao grande alongamento das células do fruto, ou seja, expansão celular com deposição de material de parede, sendo essencial o fluxo de água para o fruto neste processo, o que o faz atingir cerca de $80 \%$ do seu tamanho final, e que segundo Laviola et al. (2007) é uma fase de acúmulo de nutrientes no fruto, e ocorre em torno de 30 dias (RAMAIAH; VASUDEVA, 1969; WORMER, 1964), corroborando com esta pesquisa. Neste período, ocorreu um índice pluviométrico acumulado de $207 \mathrm{~mm}$, permitindo um excedente hídrico no solo de $11 \mathrm{~mm}$ para o mês de dezembro/2007 e de $214 \mathrm{~mm}$ para o mês de janeiro/2008, favorecendo esse processo de expansão rápida do fruto (Figura 2, Tabelas 2, 3 e 4).
Já dos 159 aos 279 DAF ocorreu uma fase de crescimento a uma taxa menor em relação à anterior (Figura 2), considerada fase de armazenamento caracterizada pelo desaceleramento no crescimento do fruto de cafeeiro, com taxas menores de acúmulo de matéria seca (RENA et al., 1994). Nesta fase ocorre o enchimento do endosperma (granação) (LAVIOLA et al., 2007), o aumento do teor de açúcares e as alterações na coloração da casca do fruto (PUSCHMANN, 1975; RENA et al., 1994), e isto ocorre em torno de 100 dias (RAMAIAH; VASUDEVA, 1969; WORMER, 1964), corroborando com esta pesquisa. Neste período ocorreu um índice pluviométrico acumulado de $830 \mathrm{~mm}$, apresentando um excedente hídrico acumulado nos meses de fevereiro até maio de 236 mm (Tabela 4).

Tabela 4. Parâmetros do modelo de crescimento exponencial para a massa seca em função da massa fresca do fruto de cafeeiro para os 3 alinhamentos nas faces $\mathrm{N}$ e $\mathrm{S}$.

\begin{tabular}{|c|c|c|c|c|}
\hline \multirow{2}{*}{ Alinhamentos (Variáveis - Y,X) } & \multicolumn{2}{|c|}{ Parâmetros } & \multirow{2}{*}{$\chi^{2}$} & \multirow{2}{*}{$\mathbf{R}^{2}$} \\
\hline & A1 & t1 & & \\
\hline $81^{\circ}-261^{\circ}(\mathrm{MSxMF}-\mathrm{FN})$ & 0,03 & 0,42 & 0,0008 & 0,96 \\
\hline $81^{\circ}-261^{\circ}(\mathrm{MSxMF}-\mathrm{FS})$ & 0,02 & 0,34 & 0,0007 & 0,95 \\
\hline $51^{\circ}-231^{\circ}(\mathrm{MSxMF}-\mathrm{FN})$ & 0,02 & 0,35 & 0,0005 & 0,97 \\
\hline $51^{\circ}-231^{\circ}(\mathrm{MSxMF}-\mathrm{FS})$ & 0,02 & 0,36 & 0,0003 & 0,98 \\
\hline $66^{\circ}-246^{\circ}(\mathrm{MSxMF}-\mathrm{FN})$ & 0,02 & 0,36 & 0,0005 & 0,96 \\
\hline $66^{\circ}-246^{\circ}(\mathrm{MSxMF}-\mathrm{FS})$ & 0,01 & 0,33 & 0,0005 & 0,96 \\
\hline
\end{tabular}

$\mathrm{MF}=$ massa fresca; $\mathrm{MS}=$ massa seca; $\mathrm{FN}=$ face norte; $\mathrm{FS}=$ face sul.

Segundo Laviola (2007), os incrementos mais significativos de acúmulo de matéria seca são nos estádios de expansão rápida e granação, sendo maior neste último. Nestas duas últimas fases de crescimento do fruto, exponencial e linear, ocorreram períodos que não apresentaram déficit hídrico no solo, representando $13 \%$ e 59\% do índice pluviométrico ocorridos nos anos de 2007 e 2008, respectivamente (Tabelas 2, 3 e 4).

Após a última fase de crescimento, depois dos 279 DAF, ocorre a fase regressiva, na qual a taxa de crescimento é inibida e o fruto desidratase na própria planta perdendo massa, momento da maturação seca.
Houve uma demora considerável no aparecimento do fruto (chumbinho), pois segundo Wormer (1964) o aparecimento do fruto ocorre entre 6 a 8 semanas após o florescimento. Neste estudo ocorreu depois de 14 semanas, o que é devido ao déficit hídrico no solo (estiagem) ocorrido no período, dificultando o aparecimento do mesmo e abortando muitas flores. Ainda, segundo o mesmo autor, o período de maior crescimento do fruto (fase exponencial) termina quando o fruto tem 17 semanas de idade, o que nesta pesquisa ocorreu com 22 semanas, conforme a (Figura 2). Quando o fruto está no final da fase linear, ele atinge seu tamanho final (maturação) ocorrendo logo após uma pequena perda de massa 
fresca, pois a massa fresca aumenta pouco e a massa seca aumenta mais, até o momento em que começa a diminuir também a massa seca devido à perda d'água (Figura 2).

As diferenças na duração de cada fase são variáveis e dependentes das cultivares e condições climáticas (SALAZAR-GUTIÉRREZ et al., 1994), pois a temperatura possui influência direta sobre processos regulatórios da planta (LARCHER, 2004) e influencia na absorção e partição de nutrientes para o fruto do cafeeiro (LAVIOLA, 2007).

A massa fresca e seca do fruto aumenta consideravelmente até o processo de maturação do fruto, em torno de 121 e $106 \%$, respectivamente (WORMER, 1964), o que nesta pesquisa o aumento percentual para a massa fresca foi de 79,78 e $80 \%$, respectivamente para os alinhamentos $81-261^{\circ}, 51-$ $231^{\circ}$ e $66-246^{\circ}$; e para a massa seca foi de 87,84 e $84 \%$, respectivamente. Com relação aos valores médios de massa fresca e seca, o alinhamento 81$261^{\circ}$ foi o que apresentou os maiores valores em relação aos demais alinhamentos.

É evidente que o componente ambiental tem grande influência no crescimento do fruto do cafeeiro, pois este é bastante influenciado por variações de temperatura, luminosidade, espaçamento de plantio, umidade, adubação, entre outras (EIRA AGUIAR et al., 2004). Considerando que o fruto teve um menor percentual de crescimento e também demorou mais para atingir a maturação em relação à citação, isso se deve ao déficit hídrico associado às temperaturas do ar ocorridas no período. De acordo com Camargo (1985) em regiões onde ocorrem temperaturas acima de $30{ }^{\circ} \mathrm{C}$, por um período prolongado e freqüente, na fase de florescimento, provocam abortamento dos botões florais; enquanto que em regiões com temperatura média anual de $23{ }^{\circ} \mathrm{C}$, o desenvolvimento e maturação do fruto são acelerados, acarretando perdas freqüentes na qualidade, desde que os outros fatores estejam adequados. Neste estudo, a temperatura do ar média do período foi de $23,6{ }^{\circ} \mathrm{C}$, sendo que a maturação não foi acelerada em função do estresse hídrico na fase logarítmica (florada-chumbinho). O amadurecimento do fruto em temperaturas menores é retardado pelo fato das plantas requererem maior temperatura para este processo do que o necessário para o crescimento das partes vegetativas da planta (LARCHER, 2004). Segundo Rena e Maestri (1985) a temperatura ótima para assimilação de $\mathrm{CO}_{2}$ pelo cafeeiro varia de 20 a $30^{\circ} \mathrm{C}$.

Observando as (Tabelas 2 e 3), o alinhamento 81-261 ${ }^{\circ}$ apresentou um valor médio de massa fresca e seca superior aos alinhamentos $51-231^{\circ} \mathrm{e}$ $66-246^{\circ}$, mas sem diferença significativa. Notase que o coeficiente de variação $(\mathrm{CV})$ apresentase maior principalmente na fase logarítmica e na fase exponencial de crescimento do fruto, demonstrando uma grande variabilidade no comportamento do crescimento do fruto em cada parcela analisada. O período de crescimento do fruto de cafeeiro compreendeu 197 dias, desde a florada até a maturação (seco). Observa-se ainda, que o alinhamento $81-261^{\circ}$ foi o que apresentou na média um maior crescimento do fruto, seguido dos alinhamentos $51-231^{\circ}$ e $66-246^{\circ}$.

Dos 96 aos 126 DAF (fase logarítmica) o ganho médio de massa fresca para a face $\mathrm{N}$ foi de 2,57, 2,27 e $2,33 \mathrm{mg} /$ dia, respectivamente para os alinhamentos $81-261^{\circ}, 51-231^{\circ}$ e $66-246^{\circ}$, enquanto que na face $\mathrm{S}$ foi de 2,20, 2,47 e 2,30 mg/dia, respectivamente. Para a massa seca na face $\mathrm{N}$, o ganho médio foi de $0,53,0,47$ e $0,50 \mathrm{mg} /$ dia, respectivamente para os alinhamentos $81-261^{\circ}, 51-231^{\circ}$ e $66-246^{\circ}$, enquanto que na face $\mathrm{S}$ foi de $0,43,0,53$ e $0,50 \mathrm{mg} / \mathrm{dia}$, respectivamente.

Dos 126 até os 158 DAF (fase exponencial) o ganho médio de massa fresca para a face $\mathrm{N}$ foi de 22,56, 22,59 e $17,59 \mathrm{mg} /$ dia, respectivamente para os alinhamentos $81-261^{\circ}, 51-231^{\circ}$ e $66-246^{\circ}$. Já na face $\mathrm{S}$, o ganho médio foi de 23,13, 19,63 e 20,47 $\mathrm{mg} /$ dia, respectivamente para os alinhamentos 81 $261^{\circ}, 51-231^{\circ}$ e $66-246^{\circ}$. Para a massa seca na face $\mathrm{N}$, o ganho médio foi de $3,56,3,91$ e $2,87 \mathrm{mg} / \mathrm{dia}$, 
respectivamente para os alinhamentos $81-261^{\circ}$, $51-231^{\circ}$ e $66-246^{\circ}$, enquanto que na face $\mathrm{S}$ foi de $3,69,3,22$ e $3,34 \mathrm{mg} / \mathrm{dia}$, respectivamente para os alinhamentos $81-261^{\circ}, 51-231^{\circ}$ e $66-246^{\circ}$.

Dos 158 até os 279 DAF (fase linear) o ganho médio de massa fresca para a face $\mathrm{N}$ foi de 59,19 , 56,02 e $55,26 \mathrm{mg} / \mathrm{dia}$, respectivamente para os alinhamentos $81-261^{\circ}, 51-231^{\circ}$ e 66-246 . Já na face $\mathrm{S}$, o ganho médio foi de 57,13, 53,69 e 53,85 $\mathrm{mg} / \mathrm{dia}$, respectivamente para os alinhamentos 81 $261^{\circ}, 51-231^{\circ}$ e $66-246^{\circ}$. Para a massa seca na face $\mathrm{N}$, o ganho médio foi de 14,90, 13,02 e 12,75 mg/ dia, respectivamente para os alinhamentos $81-261^{\circ}$, $51-231^{\circ}$ e $66-246^{\circ}$, enquanto que na face $\mathrm{S}$ foi de $12,36,11,96$ e $12,44 \mathrm{mg} /$ dia, respectivamente para os alinhamentos $81-261^{\circ}, 51-231^{\circ}$ e $66-246^{\circ}$.

Técnicas de ajuste de curva vêm sendo utilizadas no equacionamento dos mais distintos fenômenos. No entanto, a escolha inadequada de um modelo muitas vezes proporciona um ajuste aceitável matematicamente, mas não refletindo a realidade (GUIMARÃES, 2001). Para a realização de um ajuste é necessário optar por um modelo que represente a distribuição dos dados obtidos experimentalmente, e que possua parâmetros que expressem o significado físico.

A (Figura 3) apresenta a curva exponencial que foi ajustada para a correlação entre massa fresca e seca do fruto do cafeeiro, e a (Tabela 4) apresenta os parâmetros do modelo. Neste modelo, o $y$ e o $x$ correspondem à massa seca e fresca, respectivamente. Nota-se que o parâmetro $A 1$ indica o valor mínimo e $t 1$ indica o valor máximo que a massa seca atinge em $\mathrm{g}$.

Pela (Figura 4), curva sigmoidal de crescimento, e (Tabela 5), que apresenta os parâmetros obtidos neste modelo, pode-se estimar a massa fresca e/ou seca do fruto de cafeeiro. O $y$ corresponde a massa fresca e/ou seca, e o $x$ os dias após o florescimento.
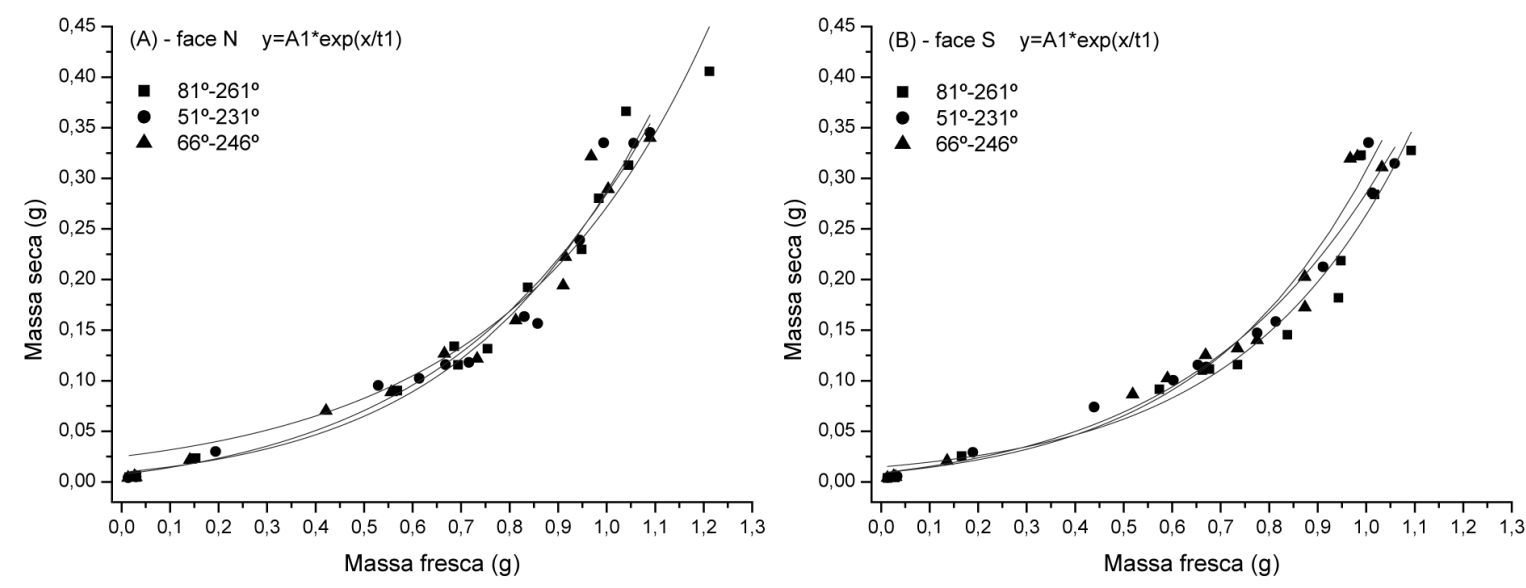

Figura 3. Curva exponencial de crescimento do fruto seco versus fruto para as faces norte (A) e sul (B) de cafeeiro cv. Obatã IAC 1669-20. 


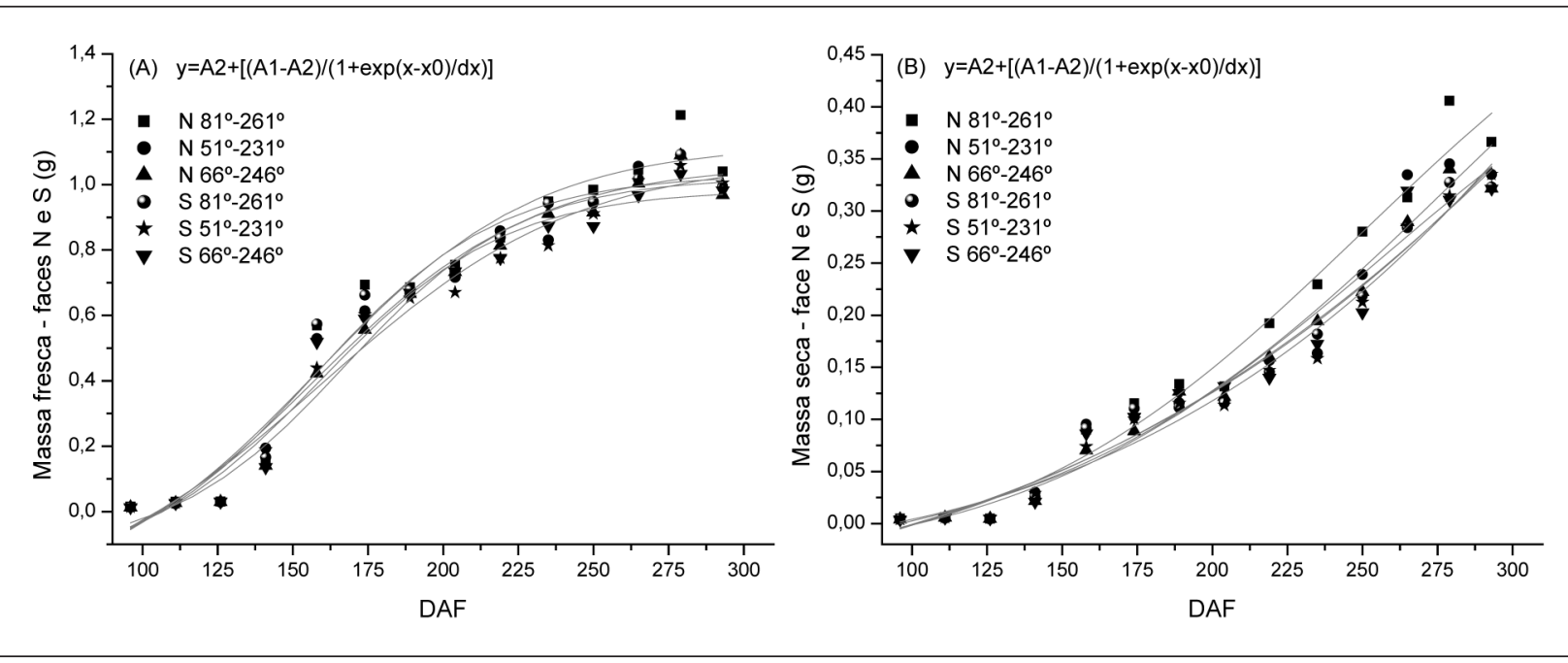

Figura 4. Curva sigmoidal de crescimento do fruto fresco (A) e seco (B) de cafeeiro cv. Obatã IAC 1669-20.

Tabela 5. Parâmetros do modelo sigmoidal de crescimento (Boltzmann) para a matéria fresca (MF) e seca (MS) do fruto de cafeeiro em função dos dias após o florescimento (DAF) para os 3 alinhamentos nas faces $\mathrm{N}$ e $\mathrm{S}$.

\begin{tabular}{|c|c|c|c|c|c|c|c|c|c|c|c|c|c|}
\hline \multirow{2}{*}{$\begin{array}{c}\text { Variáveis } \\
\text { YxX }\end{array}$} & \multirow{2}{*}{$\begin{array}{l}\text { Parâ- } \\
\text { metro }\end{array}$} & \multicolumn{2}{|c|}{$81^{\circ}-261^{\circ}$} & \multirow{2}{*}{$\chi^{2}$} & \multirow{2}{*}{$\mathbf{R}^{2}$} & \multicolumn{2}{|c|}{$51^{\circ}-231^{\circ}$} & \multirow[b]{2}{*}{$\chi^{2}$} & \multirow{2}{*}{$\mathbf{R}^{2}$} & \multicolumn{2}{|c|}{$66^{\circ}-246^{\circ}$} & \multirow[b]{2}{*}{$\chi^{2}$} & \multirow{2}{*}{$\mathbf{R}^{2}$} \\
\hline & & FN & FS & & & FN & FS & & & FN & FS & & \\
\hline \multirow[t]{4}{*}{ MFxDAF } & A1 & $-0,27$ & $-0,20$ & $\mathrm{~N}$ & $\mathrm{~N}$ & $-0,26$ & $-0,30$ & $\mathrm{~N}$ & $\mathrm{~N}$ & $-0,13$ & $-0,17$ & $\mathrm{~N}$ & $\mathrm{~N}$ \\
\hline & $\mathrm{A} 2$ & 1,12 & 1,03 & 0,009 & 0,96 & 1,06 & 1,08 & 0,006 & 0,97 & 1,02 & 0,98 & 0,003 & 0,98 \\
\hline & $\mathrm{x} 0$ & 157,82 & 157,01 & S & S & 157,42 & 159,48 & $\mathrm{~S}$ & S & 167,62 & 159,57 & S & S \\
\hline & $d x$ & 36,67 & 30,89 & 0,007 & 0,97 & 36,67 & 42,65 & 0,004 & 0,98 & 29,27 & 30,56 & 0,005 & 0,97 \\
\hline \multirow[t]{4}{*}{ MSxDAF } & A1 & $-0,05$ & $-0,09$ & $\mathrm{~N}$ & $\mathrm{~N}$ & $-0,04$ & $-0,06$ & $\mathrm{~N}$ & $\mathrm{~N}$ & $-0,05$ & $-0,10$ & $\mathrm{~N}$ & $\mathrm{~N}$ \\
\hline & $\mathrm{A} 2$ & 0,60 & 1,43 & 0,0006 & 0,98 & 0,69 & 1,38 & 0,0008 & 0,96 & 0,57 & 1,25 & 0,0003 & 0,98 \\
\hline & $\mathrm{x} 0$ & 249,43 & 391,11 & S & S & 278,90 & 378,18 & S & S & 259,07 & 370,99 & S & $\mathrm{S}$ \\
\hline & $\mathrm{dx}$ & 59,21 & 108,11 & 0,0004 & 0,97 & 65,22 & 92,46 & 0,0003 & 0,98 & 63,08 & 107,01 & 0,0007 & 0,96 \\
\hline
\end{tabular}

$\mathrm{DAF}=$ dia após a florada; $\mathrm{MF}=$ massa fresca; $\mathrm{MS}=$ massa seca; $\mathrm{FN}=$ face norte; $\mathrm{FS}=$ face sul. Médias seguidas da mesma letra não diferem entre si pelo teste $\mathrm{F}$ ao nível de $1 \%$ de probabilidade.

Considerando os parâmetros do modelo sigmoidal de Boltzmann utilizado, temos que: $A 1$ o valor inicial da massa fresca ou seca, $A 2$ o valor final da massa fresca ou seca, $x 0$ o valor da ordenada $(x)$ que corresponde ao valor médio da massa fresca ou seca no ponto de inflexão da curva (momento em que o fruto cresce com menor intensidade), e o $d x$ que indica o grau de aumento da massa fresca ou seca em função do tempo - dias após o florescimento (Tabela 5).

A evolução de uma curva sigmoidal apresenta três fases de crescimento: a) período juvenil, caracterizado por um rápido crescimento inicial (exponencial) com a concavidade voltada para cima; b) período retilíneo, em que a curva tende a linearidade; c) período de senescência, caracterizado por um crescimento cada vez mais insignificante, apresentando no final uma nítida tendência de concavidade voltada para baixo.

De forma prática, temos que o $A 1$ indica o momento na curva em que se iniciam os ganhos no acúmulo de matéria seca no fruto, o $A 2$ indica o momento em que o acúmulo começa a se estabilizar, o $x 0$ corresponde ao instante em que ocorreu a taxa máxima de acúmulo de matéria seca no fruto, e $d x$ indica de quanto foi esse aumento. Isto corrobora 
com o fenômeno biológico, pois segundo Laviola (2007) os parâmetros do modelo delimitam os estádios de crescimento do fruto com base na curva de matéria seca.

Analisando-se os parâmetros $\chi^{2}$ e $\mathrm{R}^{2}$ obtidos no modelo sigmoidal da Tabela 5, verifica-se que o alinhamento $66-246^{\circ}$ foi o que apresentou valores menores de $\chi^{2}$ e maiores de $\mathrm{R}^{2}$ para a face $\mathrm{N}$, enquanto que o alinhamento $51-231^{\circ}$ foi o que apresentou valores menores de $\chi^{2}$ e maiores de $\mathrm{R}^{2}$ para a face $\mathrm{S}$, para a massa fresca em função da massa seca, indicando que o modelo se ajustou melhor aos dados observados nestes alinhamentos.

Instrumentos estatísticos devem ser utilizados com rigor e precisão para decidir sobre os modelos que melhor se ajustam ao crescimento do fruto (DAMATTA; RONCHI; MAESTRI, 2007), sendo que esses modelos matemáticos gerados devem representar o comportamento biológico do crescimento do fruto. Com isso, torna-se possível realizar simulações onde situações reais podem ser representadas por essas equações obtidas, podendo auxiliar no manejo da colheita e na previsão de produção em função de variáveis meteorológicas, pois o crescimento do fruto depende não só da planta em si, mas também das condições climáticas que ela está submetida. Segundo Geromel (2006), a luz influencia no ciclo de desenvolvimento do fruto, estendendo-o e alterando o acúmulo de sacarose e as atividades das enzimas IAV, SUS e SPS, ao longo do desenvolvimento do fruto, uma vez que a sacarose desempenha papel fundamental na qualidade da bebida do café.

Muitos trabalhos utilizaram-se do modelo sigmoidal para descrever o crescimento do fruto de cafeeiro, tais como em Dancer (1964), Wormer e Njunguna (1966), Cannell (1971), Coombe (1976), Oyebade (1976), Gómez (1977), Portes e Castro Junior (1991), Rena et al. (1994), Silveira (1996), Silva e Volpe (2005), Geromel (2006), Ronchi et al. (2006), Ronchi e DaMatta (2007) e Laviola et al. (2007).

\section{Conclusões}

1 - Os três alinhamentos utilizados não apresentaram diferenças no valor médio de massa fresca e seca;

2 - A taxa de crescimento do fruto em massa fresca foi maior na fase exponencial, enquanto que na fase linear foi maior a taxa de crescimento em massa seca;

3 - O modelo sigmoidal representa biologicamente o crescimento do fruto do cafeeiro em massa fresca e seca em função dos dias após o florescimento, com três fases distintas, sendo a primeira logarítmica, a segunda exponencial e a terceira linear;

4 - A estimativa de massa seca em função da massa fresca do fruto pode ser expressa por modelo de crescimento exponencial;

5 - O modelo exponencial ajustou-se melhor aos dados do alinhamento $66-246^{\circ}$ para a face $\mathrm{N}$, e para a face $\mathrm{S}$ aos dados do alinhamento $51-231^{\circ}$, para a massa fresca em função da massa seca.

\section{Agradecimentos}

À Fundação de Amparo à Pesquisa do Estado de São Paulo (FAPESP) pela bolsa de Pós-Doutorado concedida para a realização do referido trabalho.

\section{Referências}

CAMARGO, A. P. Balanço hídrico, florescimento e necessidade de água para o cafeeiro. In: SIMPÓSIO SOBRE O MANEJO DE ÁGUA NA AGRICULTURA, 1., 1987, Campinas. Anais... Campinas: Fundação Cargill, 1987. p. 53-90.

O clima e a cafeicultura no Brasil. Informe Agropecuário, Belo Horizonte, v. 11, n. 126, p. 13-26, 1985.

CAMARGO, A. P.; CAMARGO, M. B. P. Definição e esquematização das fases fenológicas do cafeeiro arábica nas condições tropicais do Brasil. Bragantia, Campinas, v. 60, n. 1, p. $65-68,2001$. 
CANNELL, M. G. R. Production and distribution of dry matter in trees of Coffea arabica L. in Kenya as affected by seasonal climatic differences and the presence of fruits. Annals of Applied Biology, London, v. 67, n. 1, p. 99-120, 1971.

CASTILLO, Z. J.; LOPEZ, A. R. Nota sobre el efecto de la intensidad de la luz en la floración del cafeto. Cenicafé, Chinchina, v. 17, n. 2, p. 51-60, 1966.

CHEVALIER, A. Lês caféiers du globe. III. Systematique dês caféiers. Maladies et insects nuisibles. Encyclopedie Biologique, Paris, v. 28, n. 3, p. 1-256, 1947.

COMPANHIA NACIONAL DE ABASTECIMENTO CONAB. Acompanhamento da safra brasileira: café safra 2010. Dezembro/2010. Disponíveis no site: $<$ http:// www.conab.gov.br>. Acesso em: 04 jan. 2010.

COOMBE, B. G. The development of fleshy fruits. Annual Review Plant Physiology, Palo Alto, v. 27, p. 507-528, 1976

DAMATTA, F. M.; RONCHI, C. P.; MAESTRI, M.; BARROS, R.S. Ecophysiology of coffee growth and production. Brazilian Journal of Plant Physiology, Londrina, v. 19, n. 4, p. 85-510, 2007.

DANCER, J. The growth of the cherry of Robusta coffee. I. Weight changes correlated with water availability during development. New Phytologist, New York, v. 63, n. 1, p. 34-38, 1964.

DEAN, L. A. Relatioships between rainfall and coffee yields in the Kona district, Hawai. Journal of Agriculture Research, Washington, v. 59, n. 3, p. 217-222, 1939.

EIRA AGUIAR, A. T.; GUERREIRO FILHO, O.; MALUF, M. P.; GALlO, P. B.; FAZUOLI, L. C. Caracterização de cultivares de Coffea arabica mediante utilização de descritores mínimos. Bragantia, Campinas, v. 63, n. 2, p. 179-192, 2004.

FAZUOLI, L. C.; MEDINA FILHO, H. P.; GUERREIRO FILHO, O.; GONÇALVES, W.; SILVAROLLA, M. B.; GALLO, P. B. Cultivares de café IAC apropriadas para o plantio adensado. Campinas: Instituto Agronômico, 2000. 5 p.

FAZUOLI, L. C.; VALARINI, V.; CONCEIÇÃO, A. S.; BERNARDI, J. A. Força de adesão de frutos de vários tipos de cafeeiro. In: CONGRESSO BRASILEIRO DE PESQUISAS CAFEEIRAS, 27., 2001, Uberaba. Resumos... Rio de Janeiro: MA/PROCAFÉ, 2001. p. 117-118.

GEROMEL, C. Metabolismo da sacarose em frutos do café. 2006. Tese (Doutorado em Biologia Vegetal) Universidade Estadual de Campinas, Campinas.
GÓMEZ, G. L. Influencia de los factores climáticos sobre la periodicidad de crecimiento del cafeto. Cenicafé, Chinchina, v. 28, n. 1, p. 3-17, 1977.

GUIMARÃES, P. S. Ajuste de curvas experimentais. Santa Maria: Editora UFSM, RS, 2001. 233 p.

JONES, H. G. Plants and microclimate: a quantitative approach to environmental plant physiology. Cambridge: Cambridge University Press, 1994. 428 p.

LARCHER, W. Ecofisiologia vegetal. São Carlos: RiMa, 2004. $531 \mathrm{p}$.

LAVIOLA, B. G. Alocação de fotoassimilados e nutrientes em folhas e frutos de cafeeiro em diferentes altitudes de cultivo. 2007. Tese (Doutorado em Fitotecnia) - Universidade Federal de Viçosa, Viçosa.

LAVIOLA, B. G.; MARTINEZ, H. E. P.; SALOMÃO, L. C. C.; CRUZ, C. D.; MENDONÇA, S. M. Acúmulo de nutrientes em frutos de cafeeiro em quatro altitudes de cultivo: cálcio, magnésio e enxofre. Revista Brasileira de Ciência do Solo, Campinas, v. 31, p. 1451-1462, 2007.

LEON, J.; FOURNIER, L. Crecimiento y desarrollo del fruto de Coffea arabica L. Turrialba, San Jose, v. 12, p. 65-74, 1962.

MICROCAL SOFTWARE, INC. Data analysis and technical graphics software. Origin Professional Version 6.0. Northampton, MA: Copyright $@$, 1999. 772 p.

MOENS, P. Étude ecologique du development genératif et végétatif des burgeons de Coffea canephora Pierre: l'initiation florale. Inst. Nat. Étude Agron. Congo (INEAC). 1962. (Sér. Scientifique, v. 96).

OYEBADE, T. Studies on the pattern of growth and development of Coffea canephora fruit in Nigeria. Turrialba, Costa Rica, v. 26, n. 3, p. 257-260, 1976.

PORTES, T. A.; CASTRO JUNIOR, L. G. C. Análise de crescimento de plantas: um programa computacional auxiliar. Revista Brasileira de Fisiologia Vegetal, Brasília, v. 3, n. 1, p. 53-56, 1991.

PUSCHMANN, R. Características bioquímicas do fruto do cafeeiro (Coffea arabica L.) durante a maturação. 1975. Dissertação (Mestrado em Fisiologia Vegetal) Universidade Federal de Viçosa, Viçosa.

RAMAIAH, P. K.; VASUDEVA, N. Observations on the growth of coffee berries in South India. Turrialba, Costa Rica, v. 19, p. 455-464, 1969.

RENA,A. B.; BARROS, R. S.; MAESTRI, M.; SÖDAHL, M. R. Coffee. In: SCHAFFER, B.; ANDERSEN, P. C. (Ed.). Handbook of environmental physiology of fruit crops. Subtropical and tropical crops. Boca Raton: CRC Press, 1994. v. 2, p. 101-122. 
RENA, A. B.; MAESTRI, M. Ecofisiologia do cafeeiro. In: CASTRO, P. R. C.; FERREIRA, S. O.; YAMADA, T. Ecofisiologia da produção agrícola. Piracicaba: Associação Brasileira para Pesquisa da Potassa e do Fosfato, 1987. p. 119-147.

Fisiologia do cafeeiro. In: RENA, A. B.; MALAVOLTA, E.; ROCHA, M.; YAMADA, T. Cultura do cafeeiro: fatores que afetam a produtividade. Piracicaba: POTAFOS, 1986. p. 13-85.

. Fisiologia do cafeeiro. Informe Agropecuário, v. 11, p. 26-40, 1985.

RONCHI, C. P.; DAMATTA, F. M.; BATISTA, K. D.; MORAES, G. A. B. K.; LOUREIRO, M. E.; DUCATTI, C. Growth and photosynthetic down-regulation in Coffea arabica in response to restricting root volume. Functional Plant Biology, v. 33, n. 1, p. 1013-1023, 2006.

RONCHI, C. P.; DAMATTA, F. M. Aspectos fisiológicos do café conilon. In: FERRÃO, R. G., FONSECA, A. F. A., BRAGANÇA, S. M., FERRÃO, M. A. G., DE MUNER, L. H. (Ed.). Café conilon. Vitória: Seag/Incaper, 2007. p. 95-115.
SALAZAR-GUTIÉRREZ,M.R.;CHAVES-CÓRDOBA, B.; RIANO-HERRERA, N. M.; ARCILA- PULGARIN, J.; JARAMILLO-ROBLEDO. Crecimiento del fruto de café Coffea arabica L. var. Colombia. Cenicafe, San Jose, v. 45, n. 2, p. 41-50, 1994.

SILVA, W. J.; VOLPE, C. A. Crescimento do fruto do cafeeiro (Coffea arabica L.) cv. Acaiá, CP 474/19 e suas relações com variáveis meteorológicas em dois sistemas de plantio, no cerrado de Uberaba-MG. Revista Brasileira de Agrometeorologia, Piracicaba, v. 13, n. 2, p. 292-302, 2005.

SILVEIRA, J. S. M. Irrigação em café: café conilon irrigado no Espírito Santo. In: SIMPÓSIO ESTADUAL DO CAFÉ, 1., 1996, Vitória. Proceedings... Vitória: CETCAF, 1996. p. 66-83.

WORMER, T. M. The growth of coffee berry. Annals of Botany, London, v. 28, n. 1, p. 47-55, 1964.

WORMER, T. M.; GITUANJA, J. Flower initiation and flowering of Coffea Arabica L. in Kenya. Experimental Agriculture, Cambridge, v. 6, n. 2, p. 157-170, 1970.

WORMER, T. M.; NJUNGUNA, S. G. Bean size and shape as quality factors in Kenya Coffee. Kenya Coffee, Nairobi, v. 31, n. 366, p. 397-405, 1966. 
